\footnotetext{
${ }^{4} J$. R. Owers-Bradley, A. L. Thomson, and M. G。 Richards, J. Phys. (Paris), Colloq. 39, C6-298 (1978).

${ }^{5}$ K. Carneiro, W. D. Ellenson, L。 Passell, J. P. McTague, and H. Taub, Phys. Rev. Lett. 37, 1695 (1976); M. Nielsen, J. P. McTague, and W. D. Ellenson, J. Phys. (Paris), Colloq. 38, C4-10 (1977) .

${ }^{6}$ M. Bretz, Phys. Rev. Lett. 38, 501 (1977).

${ }^{7}$ Grafoil, graphite foam, and ZYX graphite are products of the Union Carbide Corporation.

${ }^{8} \mathrm{Bretz}$ 's (Ref. 6) and our analyses follow that of F. L. Lederman, M. B. Salamon, and L. W. Shacklette, Phys. Rev. B $\underline{9}, 2981$ (1974). The measured data, $C^{ \pm}$, are assumed to obey $C^{ \pm}-c t=\left[A^{ \pm}\left(|t|^{-\alpha}-1\right) / \alpha\right]+B^{ \pm}$, where (+), (-) correspond to $\boldsymbol{T}>\boldsymbol{T}_{c}$ or $\boldsymbol{T}<\boldsymbol{T}_{c} ; \alpha^{+}=\alpha^{-}=\alpha$; and $c, A^{ \pm}, B^{ \pm}$, and $\alpha$ are constants. Algebraic manipulation eliminates $\alpha$. It is then possible to determine $c, A^{+} / A^{-}, B^{-} A^{+} / A^{-}-B^{+}$, and $T_{c}$, and construct a "merged" specific heat where the points below and above $T_{c}$ now fall on a common curve as a function of $|t|$. See Ref. 6 for details.

${ }^{9}$ R. Zwanzig and J. D. Ramshaw, J. Phys. A 10,65 (1977), $\alpha \cong 0.296 \pm 0.002$; T. de Neef and I. G. Entig, J.Phys. A 10,801 (1977), $\alpha \cong 0.42 \pm 0.05$; M. P. M. den Nijs, to be published; B. Nienhuis, A. N. Berker, E. K. Riedel, and M. Schick, Phys. Rev. Lett. $\underline{43}, 737$ (1979), $\alpha=1 / 3$.
}

\author{
${ }^{10}$ S. Alexander, Phys. Lett. $54 \mathrm{~A}, 353$ (1975). \\ ${ }^{11}$ E. Domany, M. Schick, and J. S. Walker, Phys. \\ Rev. Lett. 38, 1148 (1977). The honeycomb lattice is \\ discussed by E. Domany and M. Schick, Phys. Rev. B \\ (to be published). \\ ${ }^{12}$ E. Domany, M. Schick, J. S. Walker, and R。B。 \\ Griffiths, Phys. Rev. B 18, 2209 (1978). \\ ${ }^{13}$ M. Bienfait, J. G. Dash, and J. Stoltenberg, to be \\ published. \\ ${ }^{14}$ S. B. Crary and O. E. Vilches, Phys. Rev. Lett. $\underline{38}$, \\ 973 (1977); C. M. Koutsogeorgis and J. G. Daunt, J. \\ Phys。(Paris), Colloq. 39, C6-308 (1978). \\ ${ }^{15}$ S. Fain, Jr., and M. D. Chinn, J. Phys. (Paris), \\ Colloq. 38, C4-99 (1977). \\ ${ }^{16}$ G. L. Pollack, Rev. Mod. Phys. 36, 748 (1964). \\ ${ }^{17} \mathrm{~N}$. Berker, private communication. We thank him \\ for his finite-size $q$-state Potts triangular lattice heat \\ capacity tables. \\ ${ }^{18} \mathrm{~J}$. Rehr and M. Tejwani, Phys. Rev. B $\underline{20}, 345$ (1979). \\ ${ }^{19} \mathrm{He} / \mathrm{Kr} /$ Grafoil measurements, O. Ferreira, Ph.D. \\ dissertation, Instituto de Fisica, Universidade de \\ Campinas, Campinas, Brasil, 1978 (unpublished); ${ }^{4} \mathrm{He}$ \\ /Kr/graphite foam measurements, M. J. Tejwani, \\ Ph.D. thesis, University of Washington, 1979 (unpub- \\ lished), and M. Tejwani, O. Ferreira, and O. E. Vilches, \\ to be published.
}

\title{
Site-Selective Doping of Compound Semiconductors by Ion Implantation of Radioactive Nuclei
}

\author{
G. Weyer, J. W. Petersen, S. Damgaard, and H. L. Nielsen \\ Institute of Physics, University of Aarhus, DK-8000 Aarhus C, Denmark \\ and \\ J. Heinemeier ${ }^{(a)}$ \\ EP Division, CERN, Geneva, Switzerland, and the ISOLDE Collaboration, CERN, Geneva, Switzerland
}

(Received 15 October 1979)

\begin{abstract}
Sn impurity atoms have been selectively inserted on the two different substitutional lattice sites in the III-V semiconductors GaP, GaAs, GaSb, InP, InAs, and InSb. Radioactive ${ }^{119} \mathrm{In}^{+}$and ${ }^{119} \mathrm{Sb}^{+}$ions which decay to the Mössbauer state of ${ }^{119} \mathrm{Sn}$ have been implanted. From the isomer shifts determined in Mössbauer-emission experiments it is concluded that the implanted In and $\mathrm{Sb}$ ions selectively populate $\mathrm{II}$ and $\mathrm{V}$ sites, respectively.
\end{abstract}

Doping of semiconductors by ion-implantation techniques is widely utilized for technological as well as for research applications. Mainly ions of the desired dopant elements have been implanted. Here we report on a new method, particularly attractive for doping of compound semiconductors. The radioactive ions that are implanted decay to the desired dopant element. As shown in the present investigation, this method enables a selection of the lattice site for, e.g., amphoteric dopants like Sn in III-V semiconductors. Although technological applications might be hampered by the relatively large amounts of ra- dioactivity needed, the method has promising aspects for microscopic investigations of dopant properties.

Radioactive ${ }^{119} \mathrm{Sb}^{+}$ions were implanted at room temperature at an energy of $80 \mathrm{keV}$ to a dose of $\sim 10^{13}$ atoms $/ \mathrm{cm}^{2}$ with an isotope separator. The ${ }^{119} \mathrm{Sb}$ activity was obtained from a bombardment of natural tin with $20-\mathrm{MeV} \alpha$ particles by a procedure described previously. ${ }^{1}$ The radioactive ${ }^{119} \mathrm{In}^{+}$ions were obtained as proton induced fission products in a uranium carbide target irradiated by $600-\mathrm{MeV}$ protons from the CERN synchrocyclotron. Following on-line mass separation in 


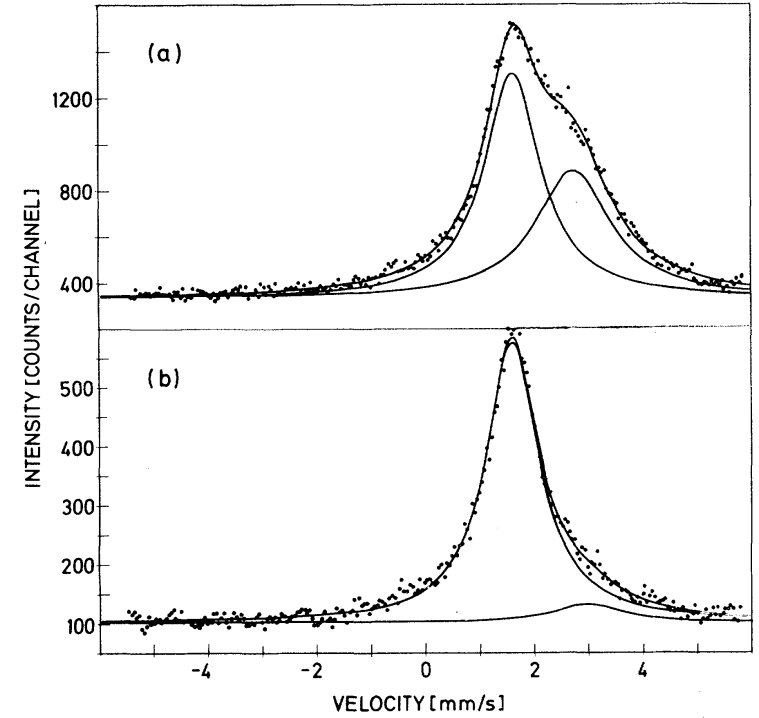

FIG. 1. Mössbauer emission spectra from ${ }^{119}$ In in InP measured at liquid-nitrogen temperature. A $\mathrm{CaSnO}_{3}$ resonance counter was used. An unannealed sample (spectrum $a$ ) and a sample annealed at $270^{\circ} \mathrm{C}$ for 1 min (spectrum $b$ ) have been measured.

the ISOLDE mass separator, ${ }^{2}$ the $60-\mathrm{keV}$ ions were implanted at a rate of $\sim 5 \times 10^{8}$ ions/s for $\sim 4$ min. All implanted semiconductor single crystals were $n$-type material.

The experimental problems of measuring Mössbauer emission spectra for the weak ${ }^{119} \mathrm{Sb}$ sources $\left(T_{1 / 2}=38 \mathrm{~h}\right.$, source strength $\left.\sim 1 \mu \mathrm{Ci}\right)$ and the strong ${ }^{119}$ In sources $\left(T_{1 / 2}=2.1 \mathrm{~min}\right.$, source strength $\sim 10 \mathrm{mCi}$ ) were solved by the application of fast resonance detectors. ${ }^{3}$ Extreme source strength was essential for the ${ }^{119}$ In experiments since spectra had to be measured within a few minutes. Details of the experimental procedures will be published elsewhere.

Two Mössbauer spectra measured at $\sim 77 \mathrm{~K}$ from implantations of ${ }^{119}$ In into InP at room temperature are shown in Fig. 1. Spectrum $a$ was measured within 4 min after the implantation, spectrum $b$ within 4 min after an annealing of the sample for $1 \mathrm{~min}$ at $270^{\circ} \mathrm{C}$ subsequent to the implantation. The spectra have been analyzed in terms of two independent emission lines as indicated in the figure. The broadened line at $\delta=2.8$ $\mathrm{mm} / \mathrm{s}$ is seen to decrease after the annealing of the sample. This is attributed to the annealing of a parent ${ }^{119} \mathrm{In}$-vacancy complex, from which this line originated. Similar impurity defects have been found in most room-temperature implanted compound semiconductors. Their Mössbauer parameters and annealing properties will

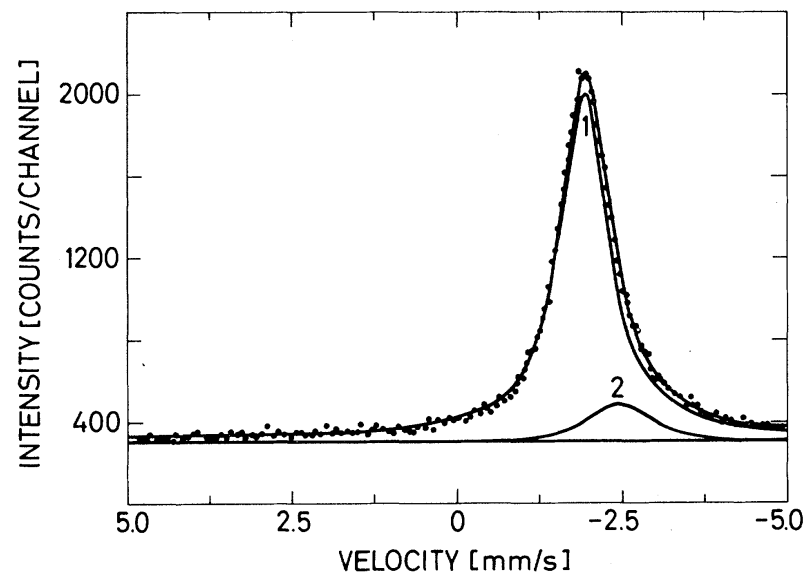

FIG. 2. Mössbauer emission spectrum from ${ }^{119} \mathrm{Sb}$ in InP (annealed at $350^{\circ} \mathrm{C}$ for $20 \mathrm{~min}$ ) measured at liquid-nitrogen temperature with a $\mathrm{CaSnO}_{3}$ resonance counter.

be reported elsewhere. Since the complex defects annealed between 200 and $400^{\circ} \mathrm{C}$ for all implanted semiconductors, predominantly single lines were left over in all spectra after an appropriate annealing. Such a spectrum for an $\mathrm{Sb}$ implantation in InP is shown in Fig. 2. The single emission lines were in all cases nearly unbroadened $(\$ 10 \%)$ indicating that the implanted atoms are in locally undisturbed cubic surroundings.

Figure 3 displays the isomer shifts of the single lines from annealed samples versus the lattice constants of the host materials together with the values for substitutional $\mathrm{Sn}$ in group-IV elements. While the isomer shifts from the Sb implantation in the III-V compounds fall on the line for group-IV elements, the values from the In implantations are systematically lower. Substitutional Sn in group-IV elements is known to have electronic structures similar to those of the host materials due to a redistribution of the Sn valence electrons according to the bond character of the hosts. ${ }^{4}$ If an analogous redistribution is assumed for the Sn impurities in III-V hosts, the difference in isomer shifts for In and Sb implantations, respectively, can be attributed to the population of the two different substitutional lattice sites in these compounds. As a consequence of the ionicity of the compound bonds in comparison to the covalent bonds of the elemental semiconductors, the electron density at the nucleus is unequal for these two sites. ${ }^{5}$ From chemical considerations, it may be expected that the electron density and hence the isomer shift is lower for the III than for the V site. Therefore, from the experimental re- 


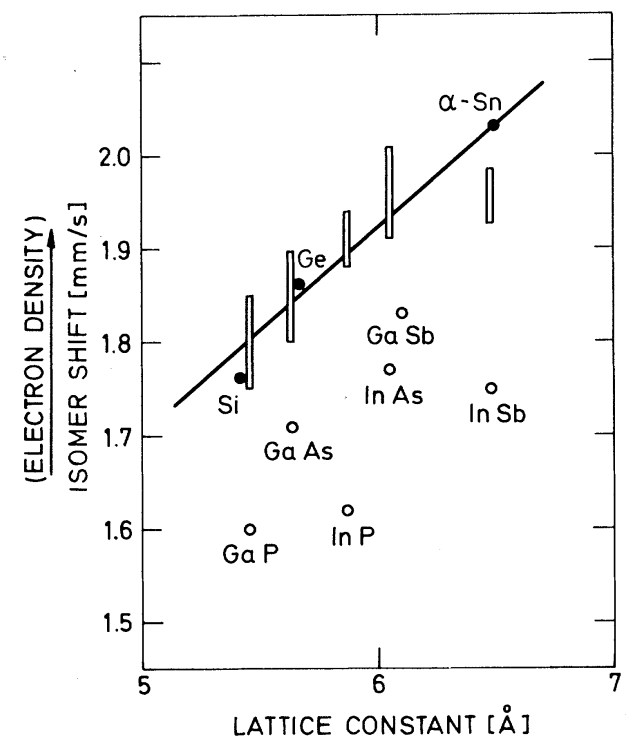

FIG. 3. Dependence of isomer shifts for Sn in elemental and compound semiconductors on the lattice constants of the host materials. Sn implantations in the elemental semiconductors are indicated by filled circles, ${ }^{119}$ In implantations by open circles, and ${ }^{119} \mathrm{Sb}$ implantations by rectangles. The isomer shifts are given relative to $\mathrm{CaSnO}_{3}$.

sults it is suggested that In and $\mathrm{Sb}$ as elements of groups III and V, respectively, preferentially occupy the III or V sites, respectively, when implanted into the III-V compound semiconductors. For the Sn atoms on the V sites the associated shallow acceptor levels can be assumed to be occupied in the $n$-type material used here. However, since these electrons are not localized on the impurity atoms, their electron densities and isomer shifts are close to those for Sn in the groupIV elements. On the other hand, Sn on III sites may or may not be positively charged according to the details of the differently doped $n$-type materials; in any case, the surplus electron will be loosely bound only. Thus a lower electron density at the nucleus should result in agreement with the experimental finding.
It is interesting to note that the above assignments are in accordance with those from Mössbauer experiments on implanted ${ }^{119 m} \mathrm{Sn}$ in $\mathrm{GaAs},{ }^{6}$ where it was concluded that $\mathrm{Sn}$ occupies the $\mathrm{Ga}$ site for annealed samples, in perfect agreement with electrical measurements. ${ }^{7}$ Obviously, for a detailed understanding of the Mössbauer parameters, quantitative model calculations are required. Nevertheless, qualitatively the basic features seem to be explained by the above considerations.

The method of site-selective doping is expected to be applicable also for other dopants in III-V or II-VI compound semiconductors.

This work has been supported by the Danish Natural Science Research Council and the Accelerator Physics Council.

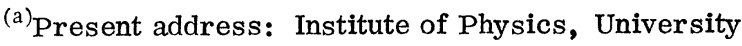
of Aarhus, DK-8000 Aarhus C, Denmark.

${ }^{1}$ G. Weyer, A. Nylandsted Larsen, N. E. Holm, and H. L. Nielsen, to be published.

${ }^{2}$ H. Ravn, L. C. Caraz, J. Denimal, E. Kugler, M. Skavestad, S. Sundell, and L. Westgaard, Nucl. Instrum. Methods 139, 267 (1976).

${ }^{3} \mathrm{G}$. Weyer, Mössbauer Effect Methodology, edited by I. J. Gruverman (Plenum, New York, 1971), Vol. 6.

${ }^{4}$ G. Weyer, A. Nylandsted Larsen, B. I. Deutch, J. U. Andersen, and E. Antoncik, Hyperfine Interact. 1, 93 (1975).

${ }^{5}$ J. P. Walter and M. L. Cohen, Phys. Rev. B $\underline{4}, 1877$ (1971).

${ }^{6}$ N. E. Holm, A. Nylandsted Larsen, B. I. Deutch, and G. Weyer, in Proceedings of the International Conference on Defects and Radiation Effects in Semiconductors, Nice, 1.978, edited by J. H. Albany (The Institute of Physics, Bristol and London, 1979), p. 573.

${ }^{7}$ R. K. Surridge, B. J. Sealy, A. D. D'Cruz, and K. G. Stephans, in Proceedings of the International Conference on Gallium Arsenide and Related Compounds, Edinburgh, 1976, edited by C. Hilsum (The Institute of Physics, Bristol and London, 1977), p. 161.
} 